\title{
Risk of biodegradable stent-induced hypergranula- tion causing re-stenosis of a gastric conduit after esophageal resection
}

Biodegradable stents are increasingly used to treat benign gastrointestinal strictures $[1,2]$. Here, we report on two patients who developed persistent vomiting after thoraco-abdominal esophageal resection for cancer and perforation. Assessments by endoscopy and imaging studies demonstrated a severe stricture of the distal gastric conduit in both patients ( Fig.1), which were thought likely to be due to low-flow ischemia of the gastric conduit. The treatment of both patients was identical. Because balloon dilation (20-mm CRE Wireguided Balloon Dilator; Boston Scientific, Galway, Ireland) had not produced an improvement in symptoms, a 10-cm long biodegradable stent (BD Ella; Ella-CS, s.r.o., Hradec Králové, Czech Republic) was placed.

Patient 1 became immediately symptomfree and remained so 12 months later. The stent was fully resorbed after 16 weeks. In contrast, patient 2 experienced persistent grade 4 dysphagia due to insufficient stent expansion ( $\bullet$ Fig. 2 a) as the stenosis was too rigid for the maximal expansive capacity of the stent. Follow-up imaging and endoscopy revealed progressive stenosis due to stent-induced hypergranulation ( Fig. 2 b and $\bullet$ Fig.3), a rare complication after the use of biodegradable stents that was also observed in some earlier studies $[1,3,4]$. Despite repeated balloon dilation to $20 \mathrm{~mm}$, the grade 4 dysphagia persisted. After 13 weeks, the stent was fully resorbed. The stenotic area was slightly enlarged compared with the situation before placement of the stent, but was still considerably smaller than the original lumen of the gastric conduit. A small, 1 to 2-cm long segment with a high-grade stenosis remained in the middle of the conduit, probably a remnant of the hypergranulation ( $\bullet$ Fig.4). Repeated balloon dilation to $20 \mathrm{~mm}$ at monthly intervals resulted in a progressive improvement over the course of 6 months ( $\bullet$ Fig.5). Thereafter, the patient was able to eat semisolid foods, consistent with grade 2 dysphagia.

Placement of biodegradable stents is an emerging and promising treatment alternative for benign esophageal strictures,

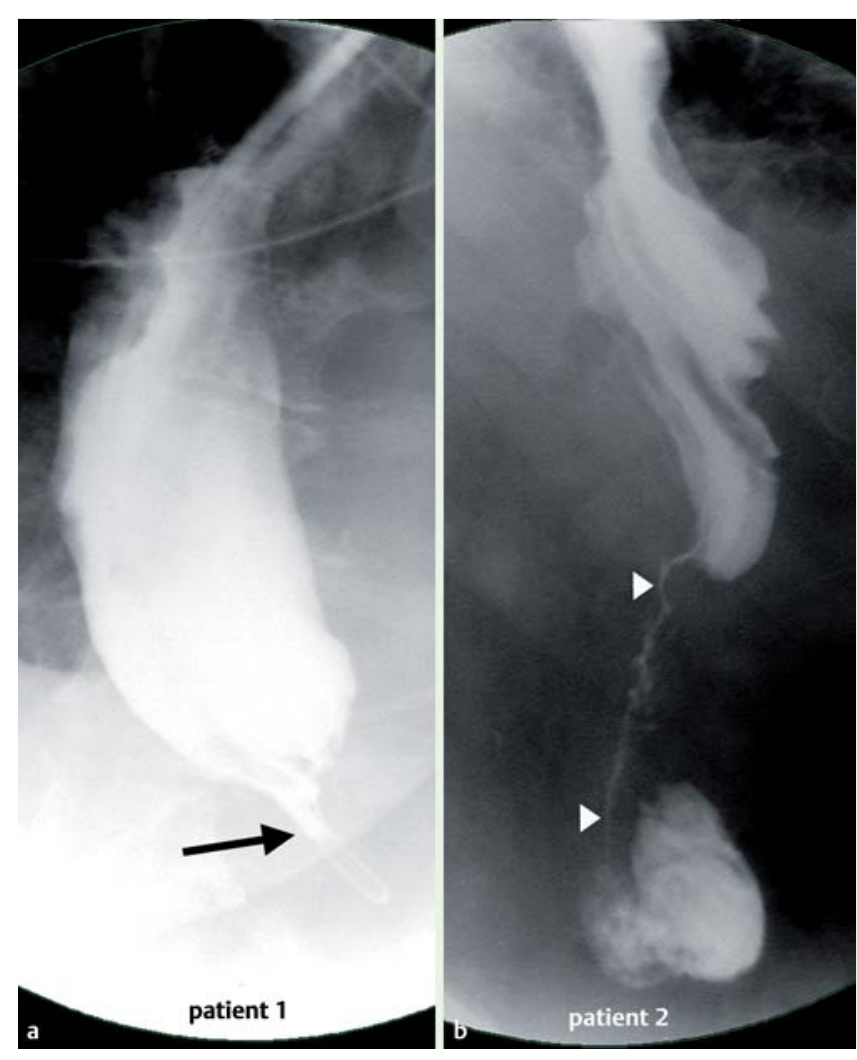

Fig. 1 Radiographic images showing: a complete stenosis within the distal gastric conduit in patient 1 (black arrow) with no further flow of the contrast agent delivered through a nasogastric tube apparent; $\mathbf{b}$ a long stenosis of the distal gastric conduit in patient 2 (white arrowheads).

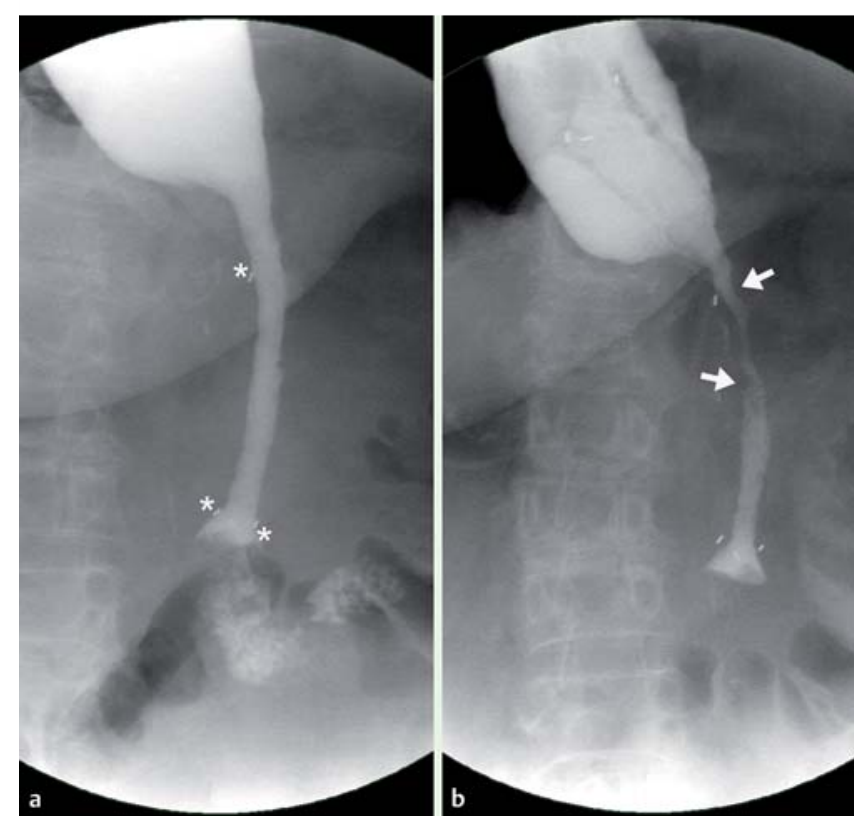

Fig. 2 Images from contrast swallow studies performed in patient 2 showing: a partial stent expansion 1 day after stent placement due to the rigidity of the stenosis (white asterisks indicate radiopaque markers); b hypergranulationinduced progressive stenosis in the stent area 3 weeks after stent placement (white arrows).

but if stenosis due to stent-induced hypergranulation occurs, significant morbidity ensues.
Endoscopy_UCTN_Code_CPL_1AH_2AD

Competing interests: None 


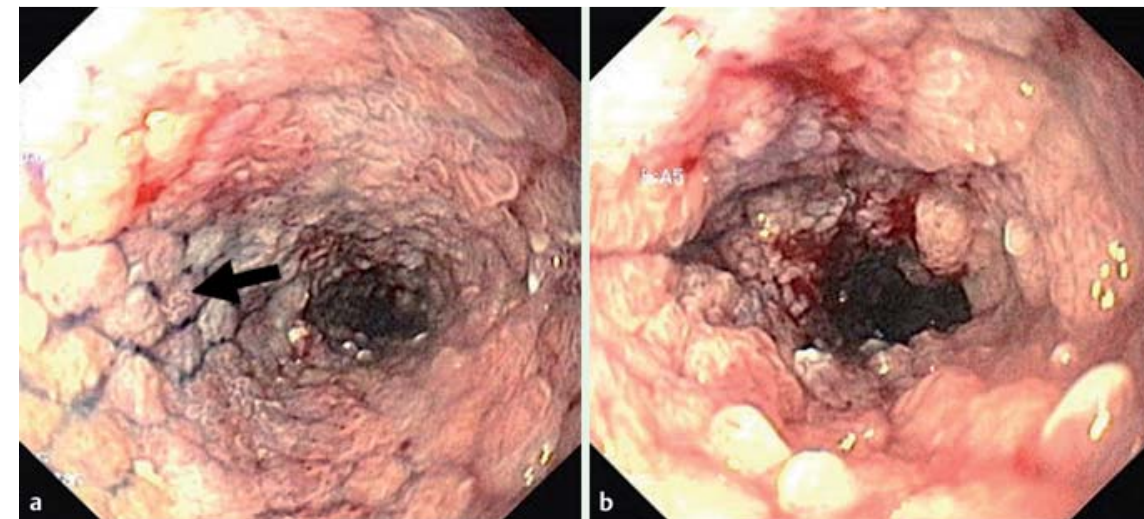

Fig. 3 Views during endoscopic examinations performed in patient 2 showing: a hypergranulation with the mesh in the middle of the stent still visible (black arrow) 3 weeks after its placement; $\mathbf{b}$ hypergranulation that completely covers the mesh in the middle of the stent 5 weeks after placement. The whole stent is still present.

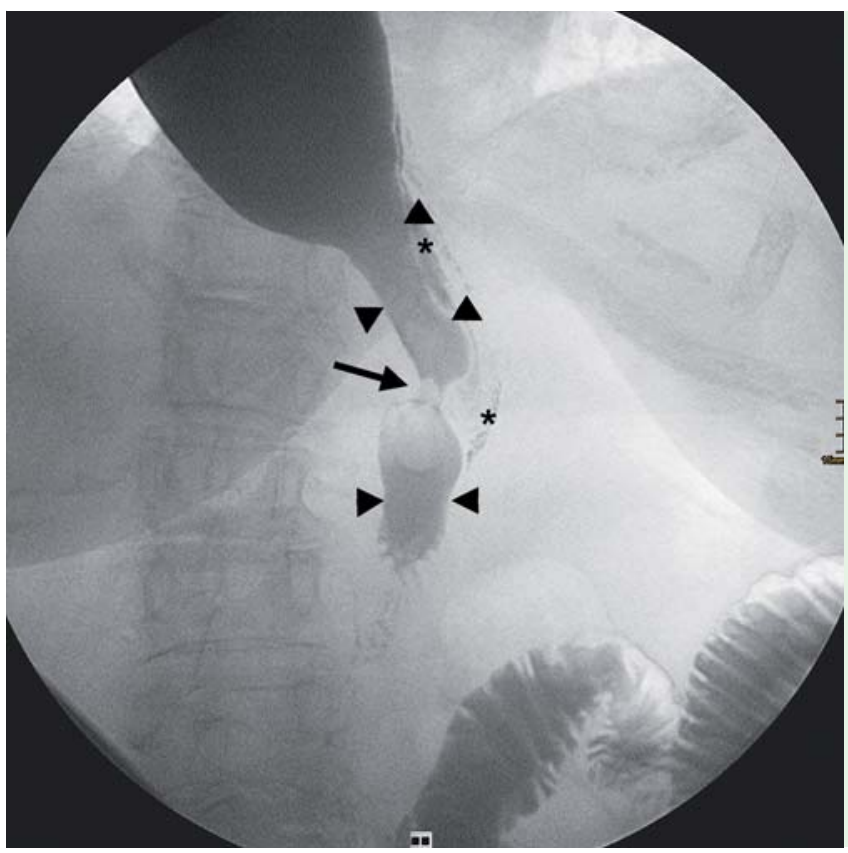

Fig. 4 Image from a contrast swallow performed before balloon dilation to $20 \mathrm{~mm}$, which reveals a long relatively mild stenosis (black arrowheads) with a short (2-cm long) markedly stenotic section (black arrow). The gastric wall contains lipiodol, which

had been previously injected to mark the stenosis $\left({ }^{*}\right)$.

\section{A. Fischer, D. Bausch, P. Baier, A. Braun, H. Richter-Schrag}

Department of General and Visceral Surgery, Albert-Ludwigs University of Freiburg, Freiburg, Germany

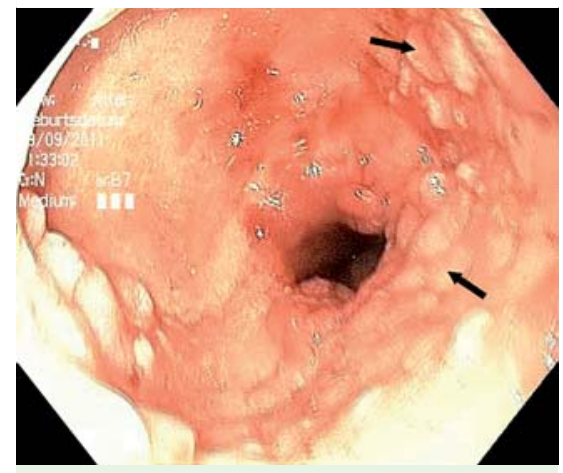

Fig. 5 View during an endoscopy performed in patient 2 some months later showing hypergranulation-induced stenosis prior to balloon dilation. Proximal to the stenosis, a raised area of granulation tissue is visible (black arrow), which was formed by the mesh of the now degraded stent.

stenoses of the small and large intestines. Endoscopy 2011; 43: 911 - 917

3 Hair CS, Devonshire DA. Severe hyperplastic tissue stenosis of a novel biodegradable esophageal stent and subsequent successful management with high-pressure balloon dilation. Endoscopy 2010; 42 (Suppl 2): E132-E133

4 Orive-Calzada A, Varez-Rubio M, Romero-Izquierdo $S$ et al. Severe epithelial hyperplasia as a complication of a novel biodegradable stent. Endoscopy 2009; 41 (Suppl 2): E137-E138

\section{Bibliography}

DOI http://dx.doi.org/

10.1055/s-0031-1291693

Endoscopy 2012; 44: E125-E126

(c) Georg Thieme Verlag KG

Stuttgart · New York

ISSN 0013-726X

\section{Corresponding author}

\section{A. Fischer, MD}

\section{Surgical Endoscopy}

Department of General and Visceral Surgery

Albert-Ludwigs University of Freiburg

Hugstetter Str. 55

Freiburg 79106

Germany

Fax: +49-761-2702543

andreas.fischer@uniklinik-freiburg.de 